\title{
Características de la Anidación de Lepidochelys olivacea (Testudinata: Cheloniidae) entre el 2010 y 2012 en Playa Tortuga Ojochal de Osa, Puntarenas, Costa Rica
}

\author{
Oscar Brenes Arias ${ }^{1 *}$, Lorena Bonilla Bonilla ${ }^{1}$, Adrián Bonilla Salazar ${ }^{1}$ \& Agustín Vega Delgado ${ }^{1}$ \\ 1. Programa de Conservación de Tortugas Marinas, Reserva Playa Tortuga, Ojochal de Osa, Puntarenas, Costa Rica; \\ oscarbreari@gmail.com, lobonilla06@gmail.com, chile87@gmail.com, agustinvega@gmail.com \\ * Correspondencia
}

Recibido 11-VIII-2014. Corregido 20-XI-2014. Aceptado 22-XII-2014.

\begin{abstract}
Features Lepidochelys olivacea Nesting (Testudinata: Cheloniidae) between 2010 and 2012 in Playa Tortuga Ojochal, Osa, Puntarenas, Costa Rica. Olive Ridley sea turtle (Lepidochelys olivacea) have been amply studied on Costa Rican beaches that experience mass nestings, "arribadas", both in their nesting behavior and other aspects of their biology; however, very little published information exists about beaches where the Olive Ridley is nesting in solitary form, especially along the Southern Pacific Coast. For this reason, the objective of this study is to describe relevant nesting aspects, such as number of nest per season and nesting sites of L. olivacea on the Tortuga Beach in the South Pacific of Costa Rica, during three nesting seasons (2010, 2011, 2012). The beach was divided into 14 sectors of $100 \mathrm{~m}$ each. Beach patrols were conducted every night from July to December (2010) and July to January $(2011,2012)$, with the purpose of observing and recording nesting behavior in the turtles as well as to protect their nests. The females observed were identified by means of metal tags applied to their rear flippers. Other data collected included: number of eggs deposited, nest location, hour, biometric data (width and length taken along the curve of the carapace), and the total of nests laid (found with or without the turtle present). After three seasons, 100 females of Olive Ridley Sea Turtle were successfully marked, and a total of 233 nests were counted. The frequency of re-nesting of an individual during a season was of every 14 to 20 days and four individuals re immigrated to nest again over the period of one year. The total population of nesting females during these three years was estimated at 117 . The peak nesting happens in September, more sea turtles visited the beach during this period in all the seasons reported. With respect to biometry, the average value of the LCC was $69.91 \pm 1.05 \mathrm{~cm}$ and the average value of the WCC was $70.476+/-1.767 \mathrm{~cm}$. A total of 18711 eggs were collected and 9858 were successfully hatched. The Tortuga Beach was proven to be a nesting beach of the species L. olivacea; the population of nesting females on the beach can be considered significantly reduced in comparison with other nesting beaches of the same species along the Southern Pacific Coast of Costa Rica. Furthermore, marking the turtles with metal tags has proven a useful tool towards the characterization of the nesting aspects of the sea turtles, such as the frequency and interval of re-nesting, and other variables such as the re immigration which requires a period of time greater than the time period of the study to date. Rev. Biol. Trop. 63 (Suppl. 1): 339-349. Epub 2015 April 01.
\end{abstract}

Key words: Sea turtle, nesting, tagging, south pacific, population.

Lepidochelys olivacea, conocida comúnmente como "tortuga lora", es una de las cinco especies de tortugas marinas que utilizan las playas de Costa Rica como sitios de anidación, se localiza exclusivamente en el litoral del Océano Pacífico del país (Chacón, Sánchez, Calvo \& Ash, 2007). Esta especie prefiere anidar en litorales e islas de barrera en regiones tropicales, cerca de las bocas de los ríos (Mortimer \& Pritchard, 2000).

Las tortugas del género Lepidochelys presentan dos tipos de comportamiento de anidación, uno es el solitario, donde los individuos arriban a las playas de manera independiente o en pequeños grupos a lo largo de la temporada de desove; el otro es masivo, conocido como 
las "arribadas", donde de una manera sincronizada miles de hembras llegan a la playa en un período de pocos días a desovar (Gulko \& Eckert, 2004).

En Costa Rica la arribada se puede observar solamente en dos playas de anidación ambas localizadas en la región norte sobre el litoral del Océano Pacífico, Playa Ostional y Nancite, las otras playas son de anidación solitaria. En la región sur de Costa Rica se destacan como playas de anidación solitaria, Ballena, Madrigal (Corcovado), Colorada en Bahía Drake, Pejeperro, Piro, Playa Sombrero, Platanares y Punta Banco (Chacón et al., 2007).

Debido a su frecuencia alta de anidación, L. olivaceae se considera la especie más abundante de tortugas marinas a nivel mundial; (Gulko \& Eckert, 2004), pero a pesar de su relativa abundancia, las tortugas lora son afectadas por disturbios antropogénicos como la sobre-explotación ,la alteración de las áreas de anidación y la captura incidental por artes de pesca, así como por factores intrínsecos de la población como un éxito bajo de eclosión (Valverde \& Gates, 2000), por lo que sus poblaciones se han visto reducidas hasta el punto, de ser considerada una especie en peligro de extinción (Chacón et al., 2007). Estas afectaciones son más evidentes en las tortugas marinas que presentan el tipo de anidación solitaria (AbreuGrobois \& Plotkin, 2008).

En el caso específico de L. olivacea, hasta el 2001, ninguna playa de anidación solitaria a nivel del istmo centroamericano había sido monitoreada con la constancia ni el período necesario para determinar cambios en la fracción de la población de tortuga lora que visita cada sitio y poder proyectarlos hacia la población total. (Chacón \& Aráuz, 2001).

Mediante el estudio de la anidación, se puede obtener información valiosa acerca de la condición y tamaño de la población de las hembras que anidan año con año. Además de identificar los factores de riesgo puntuales en una playa específica, permitiendo registrar y observar los efectos de los mismos en la población (Chacón et al., 2007).
Desde los años setenta (Hughes \& Richard, 1974), un porcentaje alto de los estudios realizados en Costa Rica acerca de diferentes aspectos de la biología de L. olivacea han sido realizados principalmente en las playas de arribada, Nancite y Ostional (Cornelius \& Robinson, 1986; Campbell, 1998; Ballestero, Aráuz \& Rojas, 1998; Plotkin, 2010). La literatura acerca de su comportamiento reproductivo en playas de anidación solitaria en el Pacífico de Costa Rica es sumamente escasa a pesar de que en muchas de estas playas se ejecutan programas de conservación de tortugas marinas, ya sea por parte del Estado, Asociaciones Comunales u Organizaciones No Gubernamentales. Este estudio tiene como objetivo describir aspectos de la anidación registrados en las hembras de $L$. olivacea en Playa Tortuga, colectados a partir de tres temporadas de monitoreo, para brindar información científica que pueda ser utilizada en otras playas del Pacífico Sur de Costa Rica, para tomar medidas de manejo adecuadas en la conservación de la especie.

\section{MATERIALES Y MÉTODOS}

Desde el año 2010 hasta el 2012, como parte del Programa de Conservación de Tortugas Marinas del Humedal Térraba-Sierpe, personal de la Reserva Playa Tortuga ha venido realizando patrullajes nocturnos en Playa Tortuga, ubicada en Ojochal de Osa, en la provincia


9॰4’32.16” N). La playa tiene una extensión de $1.5 \mathrm{~km}$, y limita al sur con el Humedal Nacional Térraba-Sierpe y al norte con el Parque Nacional Marino Ballena. Además del Río Térraba, en Playa Tortuga corren los ríos Tortuga por el extremo norte y Balso por el extremo sur.

Distribución de la anidación: La playa fue dividida de norte a sur en 14 sectores por medio de postes de madera separados $100 \mathrm{~m}$ uno del otro. Para cada hembra o nido observado se registró el número de sector.

Se patrulló la playa de manera intensiva todas las noches en un periodo de $8 \mathrm{~h}$, cubriendo en promedio una distancia de $12 \mathrm{~km}$; se caminó 
de acuerdo al ritmo de las mareas, en media marea subiente y media bajante. En las temporadas 2011 y 2012 se trabajó en el período de julio a enero y en la temporada 2010, de julio a diciembre. Esto con el fin de observar y marcar a las hembras que arribaron a Playa Tortuga, además se censaron los rastros y las nidadas efectivas.

Se contabilizó como nidada efectiva todo nido con huevos y aquellos saqueados que presentaron evidencias de la anidación, como huevos o cascarones, o bien, si se presenció al saqueador en el acto.

Identificación de individuos y marcaje: Dado que permite identificar cada tortuga individualmente, el marcaje de las hembras que anidan es considerado una herramienta útil para describir el comportamiento de la anidación (Eckert \& Beggs, 2006).

Se utilizaron marcas National modelo 681 de Inconel, cada una presentaba un código numérico único en una de sus caras y la leyenda rptojochal@gmail en la otra. Se utilizaron aplicadores específicos para el modelo de la marca.

Con la intención de no interrumpir la anidación, una vez ubicada una tortuga se observó su comportamiento (emergiendo o regresando al mar, preparando el nido, desovando o cubriendo la cámara). La marca se aplicó después del proceso de desove, justo cuando el animal comenzaba a cubrir la cámara con arena. Los individuos que se encontraron de regreso al mar fueron marcados, independientemente de si tuvo o no una anidación efectiva.

Antes de aplicar la marca, se revisó cada aleta para comprobar si el animal ya había sido marcado anteriormente, si se observó una o varias marcas cada una se reportó como vieja y se registró la aleta además el número/código de esta. Si la marca estaba pronto a perderse esta se removió y sustituyó por una nueva, si el individuo presentaba una sola marca, se aplicaba una nueva en la aleta sin marcar.

A manera de protocolo de marcaje, siempre se inició con la aleta izquierda y luego la derecha. Se trabajó el animal colocándose atrás o al lado del mismo, nunca de frente. Se marcó en las aletas frontales entre la primera y segunda escamas partiendo desde la axila del animal, si el individuo presentaba daños en esta zona a causa de heridas o marcas previas, la marca se colocó entre la segunda y tercera escama. Antes y después de aplicar la marca cada aleta se desinfectó agregando Vanodine al $1 \%$ en agua (solución a base de yodo). Los datos registrados para cada hembra fueron, la especie, el número de marca, la posición (aleta derecha o izquierda), la hora, el número de huevos (nidadas efectivas) y la fecha. Como datos biométricos a cada tortuga se midió utilizando una cinta métrica flexible de $1.5 \mathrm{~m}$, el largo curvo del caparazón (CCL) y el ancho curvo del caparazón (ACC), según Chacón et al. (2007).

Nidos: Una vez marcada y medida la tortuga se colectaron los huevos. Su ubicación se determinó, insertando una cinta métrica flexible de $1.50 \mathrm{~m}$ dentro de la cámara de anidación mientras la tortuga desovaba, o punzando la arena con una vara de madera hasta encontrar la nidada. Los huevos fueron extraídos, contabilizados y asignados con mismo código de la madre, para posteriormente colocarlos en una bolsa plástica para transportarlos al vivero. En este sitio se hizo una copia fiel de la cámara y se sembró para ser monitoreado hasta su eclosión y la liberación al mar de los neonatos. Guantes de látex fueron utilizados durante todo el proceso de manipulación de los animales y huevos.

Análisis estadístico: Se estimo un Valor NT (número total de tortugas que desovaron) para cada temporada y para el periodo total del estudio.

Este cálculo se basó en el método de estimación indirecto, establecido por, Alvarado y Murphy (2000), donde se puede tener una idea del tamaño de la población si se conoce el número de veces en que las tortugas realmente desovaron o nidadas efectivas $(\mathrm{Ne}) \mathrm{y}$ la frecuencia de anidación promedio (cuantas veces anida una misma hembra por temporada) 
reportada para la especie (Fap) por medio de la siguiente fórmula:

$$
\mathrm{NT}=(\mathrm{Ne} / \mathrm{Fap})
$$

Con el número de tortugas marcadas por temporada (NMT) y la frecuencia de anidación estimada ( $F a e$ ), se proyectó un número de nidadas potenciales $(N P)$ cantidad máxima teórica que pudieron haber aportado los individuos con marcas, mediante la siguiente fórmula:

$$
\mathrm{NP}=(\mathrm{NMT} * \mathrm{Fae})
$$

\section{RESULTADOS}

Para la temporada 2010-2011 se marcaron en total 100 tortugas, distribuidos en 16 individuos en el 2010, 45 en el 2011 y 39 individuos en el 2012.

En cuanto a nidadas efectivas (Ne), se contabilizó un total de 233, 56 en el 2010, 98 en el 2011 y 79 en el 2012. Con los datos de frecuencia de anidación $(F a e=2)$, obtenidos y el número de tortugas marcadas $(N M T=100)$, se proyectó un valor de $N P$ total igual a 200 (Fig. 1).

Además se confirmó que las hembras marcadas aportaron un número real de nidadas (NrTM) igual a 20 en la primera temporada, 52 en la segunda y 40 en la tercera. Por lo que del total de nidadas efectivas al menos 112 fueron aportadas por las hembras identificadas (Fig. 1).

Del total de tortugas marcadas, 11 individuos anidaron dos veces en una temporada, mientras que cuatro hembras anidaron en temporadas consecutivas presentando un comportamiento de remigración anual, para el resto de individuos no fue posible determinar tendencias.

Con base en los datos de marcaje y capturas, se determinó una frecuencia de anidación (Fae) para L. olivacea en Playa Tortuga de dos veces por temporada, mismo valor que



Fig. 1. Comparación del número de nidadas reales y potenciales de las hembras de tortuga Lora que fueron marcadas del año 2010 al 2012, con respecto al número de nidadas efectivas registradas durante este mismo periodo en Playa Tortuga. $\mathrm{NrTM}=$ número de nidadas reales de tortugas marcadas; $\mathrm{Np}=$ número de nidadas potenciales; $\mathrm{Ne}=$ número de nidadas efectivas.

Fig. 1.Comparison of the number of nests and nesting potential of the Olive Ridley females marked from 2010 to 2012 , with respect to the number of registered nests during the same period on Tortuga Beach. NrTM=actual clutches number of tagged turtles; $\mathrm{Np}=$ potential number of clutches; $\mathrm{Ne}=$ effective clutches number. 
el reportado para la especie por Chacón et al. (2007). Así mismo se registró un intervalo de anidación para Playa Tortuga de 14 a 20 días, entre posturas.

Basado en las nidadas efectivas registradas $(N e=233)$ y la frecuencia de anidación reportada (Fap $=2)$, se estimó que al menos 117 hembras de L. olivacea arribaron a Playa Tortuga a lo largo de las tres temporadas, el mayor número de individuos se registró en la temporada 2011(n=49); al comparar los datos obtenidos con el número de tortugas marcadas total y por temporada se observó que en la temporada 2012 se marcó el 99\% de la población estimada y en el total de las temporadas un $86 \%$ de las tortugas se identificaron (Fig. 2).

A lo largo de las tres temporadas se colectó un total de 18711 huevos, de ellos un 56\% (10 424 huevos) pertenecen a las tortugas identificadas por marcas. La temporada en la cual se colectó la mayor cantidad de huevos fue la 2011(8 509), seguida de la temporada 2012(5 $395)$ y la temporada 2010 (4 807).

Con respecto al tamaño de las nidadas, las posturas promedio obtenidas en el 2010 y 2011 fueron de $98.10+/-4.38$ y $98.92+/-4.47$ huevos por nido, respectivamente. La temporada 2012 fue la que reportó la menor cantidad de huevos promedio por nido $94.56+/-4.05$.

Los datos biométricos arrojaron una talla de Largo Curvo del Caparazón (LCC), promedio de $69.91 \pm 1.05 \mathrm{~cm}$, con una talla mínima de $57 \mathrm{~cm}$ y una máxima registrada de $79 \mathrm{~cm}$. El Ancho Curvo del Caparazón (ACC) promedio obtenido fue de $70.476+/-1.767 \mathrm{~cm}$, con mínimo de $62 \mathrm{~cm}$ y un máximo de $79 \mathrm{~cm}$.

Entre el año 2010 y 2012 se reclutó un total de 9858 neonatos de L. olivacea en Playa Tortuga, de estos, 6031 individuos (61\% del total), fueron un aporte de las hembras identificadas.

La distribución horaria de los eventos de anidación, en Playa Tortuga mostró que, las hembras de L. olivaceae, anidan durante toda la noche, teniendo los picos más bajos de arribadas al principio de la noche de las 18 a las $20 \mathrm{~h}$, y al final de la noche de las dos hasta las seis horas. Las horas de más actividad se extienden desde las 20 hasta las $2 \mathrm{~h}$, registrando el punto máximo en el intervalo comprendido desde las cero hasta las dos horas. (Fig. 3).



Fig. 2. Cantidad de hembras marcadas y número de hembras de L. olivacea estimado para Playa Tortuga, durante tres temporadas de monitoreo. NMT=número de tortugas marcadas; NT=número de tortugas estimado.

Fig. 2. Number of females marked and the estimated number of the nesting females of L. olivacea for Tortuga beach, during three monitoring seasons.NMT=tagged turtles number; NT=estimated number of turtles. 


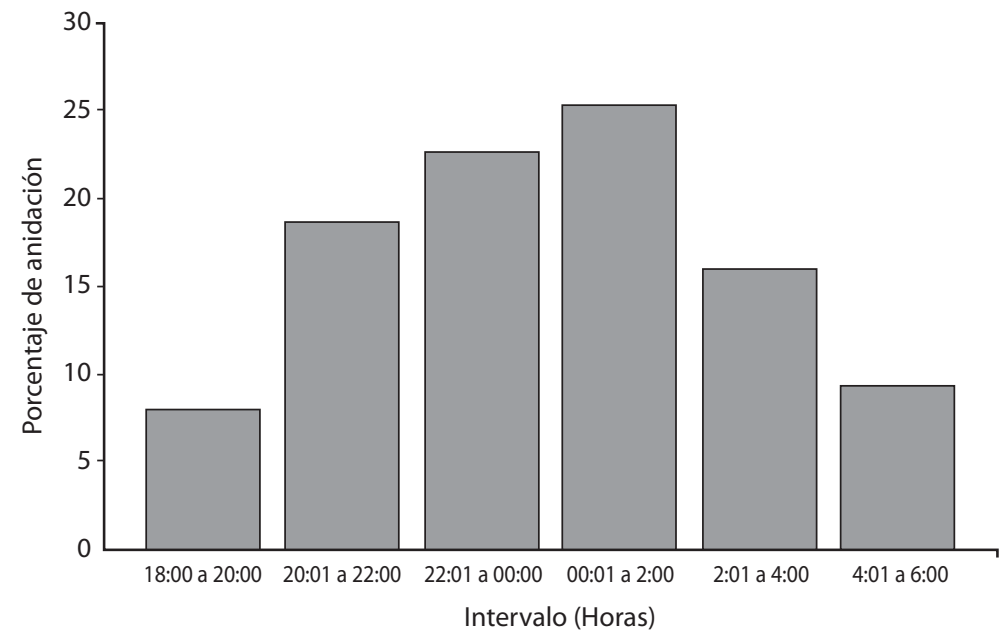

Fig. 3. Distribución horaria de los eventos de anidación registrados para L. olivacea en Playa Tortuga a partir de tres años de estudio.

Fig. 3. Time distribution of registered nesting events for L. olivacea on Tortuga Beach after 3 years of study.

Con base en datos colectados de las tres temporadas, los meses en los que se dio el mayor número de eventos de anidación en Playa Tortuga, fueron agosto y setiembre, un $50 \%$ del total de tortugas desovaron en Playa Tortuga en este periodo. A partir del mes de octubre la anidación comenzó a disminuir hasta alcanzar el menor porcentaje de visitas en diciembre con un 5\% del total. (Fig. 4).

En cuanto a la distribución espacial, las tortugas que arribaron en el 2010 anidaron por toda la playa observándose una mayor cantidad de eventos en los sectores 2 y 10, para el año 2011 se observó una disminución de la anidación a partir del sector medio de la playa (sector seis) hacia el sector 14 que demarca el extremo sur de Playa Tortuga, y en el 2012, la anidación se concentró en la primera mitad del área de muestreo entre los sectores uno y siete (Fig. 5).

\section{DISCUSIÓN}

Para el año 2007, Playa Tortuga aún no aparecía registrada como parte de las playas de anidación de tortugas marinas del Pacífico de Costa Rica (Chacón et al., 2007), por lo que con este estudio reafirmamos la actividad anidadora en esta playa.

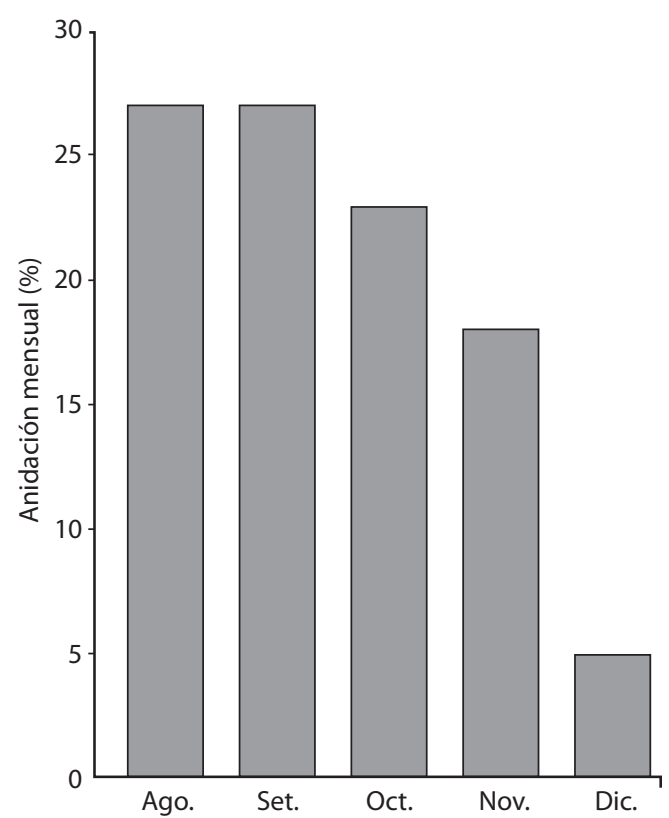

Fig. 4. Comportamiento mensual de la anidación de $L$. olivacea, observado durante el periodo 2010-2012 en Playa Tortuga.

Fig. 4. Monthly nesting behavior of L. olivacea, observed during the period of 2010-2012 on Playa Tortuga. 

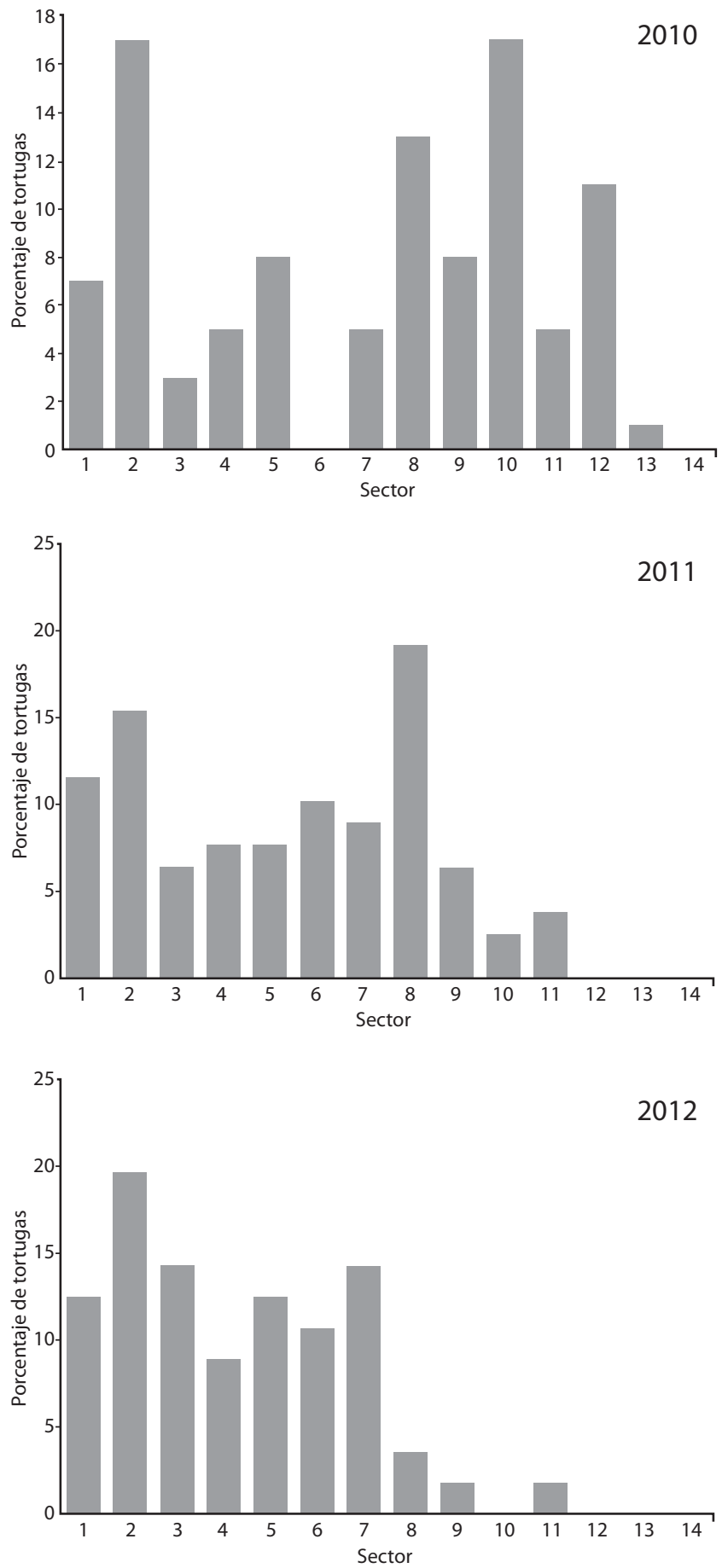

Fig. 5. Distribución espacial de la anidación registrada en playa Tortuga en cada una de las temporadas de estudio. Fig. 5. Spatial distribution of registered nesting on Tortuga beach during each season of the study. 
Basado en los datos de las tres temporadas se demuestra que Playa Tortuga, es una zona de anidación de L. olivacea en la región sur del litoral Pacífico de Costa Rica, ya que este tipo de playa con bocas de ríos cercanos representa el hábitat preferido para la especie (Mortimer $\&$ Pritchard, 2000). Se comprueba a través del comportamiento registrado por las hembras identificadas y el número de individuos estimado, que el tipo de anidación que ocurre en Playa Tortuga es del tipo solitaria.

El volumen de los eventos anidación de la tortuga lora en Playa Tortuga en comparación a otras playas del Pacífico Sur podría considerarse bajo, ya que playas como Carate, Piro, Pejeperro y Río Oro en Osa, reciben cientos de tortugas por temporada (Sánchez, 2007). El mismo autor reportó sólo para Playa Piro un total de 308 nidos de L. olivacea en la temporada 2006, 75 nidos más que el total registrado del 2010 al 2012 en Playa Tortuga.

Para una especie considerada como vulnerable, todo aporte a la población es significativo (Abreu-Grobois \& Plotkin, 2008). Este bajo número actual de hembras reproductivamente maduras en Playa Tortuga se puede atribuir a la sobre-explotación, 15 ó 20 años atrás era posible observar al menos 30 hembras de tortuga lora anidando por noche (Campos, 2010, com. pers.), número cercano al estimado actualmente para toda una temporada.

Con el esfuerzo de muestreo se ha determinado el mes setiembre como el punto más alto de la anidación en Playa Tortuga.

Las tortugas lora, anidan en la costa Pacífico Oriental entre junio y diciembre, aunque la mayor actividad ocurre en setiembre y octubre, esto puede variar de una playa a la otra, al igual que en Playa Tortuga, el mayor arribo de $L$. olivacea en Punta Ratón Honduras se da entre agosto y octubre (Chacón \& Aráuz, 2001).

El conocimiento de la anidación a lo largo de la temporada, es una herramienta importante para establecer patrones comparativos base y enfocar el esfuerzo de muestreo, por ejemplo durante los meses de más arribo de hembras, en playas donde no se pueda cubrir toda una temporada.
A pesar que se identifico el período entre las $22 \mathrm{~h}$ y las $2 \mathrm{~h}$ como el de mayor probabilidad para detectar eventos de anidación .Es recomendable en playas de poca extensión como Playa Tortuga, cubrir toda la noche, ya que se demostró un aumento en la efectividad de localización de hembras y nidos. A diferencia de las temporadas 2010 y 2011 (patrullaje dependiente de mareas), en el 2012 muchas de las noches se cubrieron totalmente, por lo que el esfuerzo se refleja en la relación de la cantidad de tortugas marcadas y la población estimada en este año (Fig. 2).

Eckert y Beggs (2006), mencionan que para establecer tendencias poblacionales de las tortugas marinas por medio del marcaje, son necesarios un mínimo de 5 a 10 años, este esfuerzo es necesario en especial para poblaciones muy pequeñas como la de Playa Tortuga. Un estudio a largo plazo en esta área se recomienda para obtener información consolidada sobre la remigración real de la especie, a cual puede anidar cada uno o dos años (Chacón et al., 2007). Sin embargo, el presente esfuerzo de muestreo ha brindado información inicial importante, como la tendencia que mostraron algunos individuos recapturados hacia una remigración anual y la definición del intervalo de reanidación y frecuencia de anidación específicos para L. olivacea en Playa Tortuga.

El marcaje en conjunto con el registro biométrico de los individuos, es importante para establecer las tasas de crecimiento de los individuos a lo largo del tiempo, ya que el registro de los cambios y análisis de las tallas de las hembras reproductivas puede ser un indicador de la calidad del hábitat y de la situación fisiológica de la población (Bolten, 2000). Con los datos obtenidos en Playa Tortuga se puede afirmar que una hembra de L. olivacea con un Largo Curvo del Caparazón (LCC) de $57 \mathrm{~cm}$ ya es un animal que ha alcanzado la madurez reproductiva. Esta información en cuanto a las tallas podría servir como base comparativa con otras playas del litoral pacífico y observar si existen variaciones de un sitio a otro. Adicionalmente, en situaciones de varamientos, muerte o captura en mar abierto, donde no se 
cuente con las herramientas necesarias para tomar muestras o ejecutar necropsias para determinar la madurez sexual del individuo, la simple colecta de las medidas del tamaño de una tortuga hembra, funcionaría como un indicio para determinar esta condición.

Con respecto a las nidadas, el número de huevos por nido registrado en playa se encuentra para bajo del promedio para la especie reportado por Chacón et al. (2007), a pesar de esto, es un número que es consistente en las temporadas, por lo que se puede reportar como específico para L. olivacea en Playa Tortuga.

El marcaje brindó información importante acerca de la influencia significativa que tuvieron las hembras marcadas con respecto al total de huevos rescatados, y el reclutamiento de neonatos a la población.

Al comparar el valor de las nidadas potenciales de las tortugas marcadas, con las nidadas efectivas registradas, se observa que al menos para las temporadas 2011-2012 se pudieron haber identificado casi en totalidad las tortugas que arribaron; este dato se refleja en valores cercanos del total de tortugas marcadas en relación a la población estimada (Fig. 1).

Con el transcurso de las temporadas se observa como la anidación de L. olivacea se ha ido concentrando del centro hacia el norte de Playa Tortuga (Fig. 5). Lo anterior se debe a que en el 2010, como producto del huracán Tomás (Chinchilla \& Naranjo, 2010), el cauce del Río Tortuga que originalmente desembocaba en la parte sur de Playa Tortuga (final del sector 14), se movió de manera progresiva erosionando la playa. Para el 2012, la desembocadura del Tortuga se desplazó hasta el sector 10, dejando solamente un kilómetro de playa disponible para anidar. Este decremento del área de anidación provoca que las tortugas se concentren en hábitat marginales, dejando poco espacio disponible, lo que podría afectar de manera directa la anidación, pues las hembras que no encuentren sitios aptos para anidar podrían verse forzadas, a volver al mar en busca de otra zona (Bolongaro, Márquez \& García, 2010).
Este desplazamiento de las tortugas hacia la parte norte de Playa Tortuga podría tener un impacto directo en la población ya que en esta zona es donde se concentra la mayor cantidad de actividades humanas. Uno de los factores de degradación de las áreas costeras es el desarrollo descontrolado, sin tomar en cuenta el riesgo futuro de la contaminación lumínica (Whinterington \& Martin, 1996; Chacón, Valerin, Cajiao, Gamboa \& Marin, 2000). Gulko y Eckert (2004), comentan que aún playas sin desarrollo pueden ser altamente alteradas por actividades recreativas, por lo que es preocupante que en esta zona de la playa, se utilice un camino con acceso directo a los vehículos y turistas a las zonas de anidación. Esto provocaría la compactación afectando la composición física de la arena, dejando de ser un sitio apto para anidar (Chacón et al., 2000).

Más allá de las amenazas en playa, aún se desconocen los alcances de los eventos de muertes masivas registrados en enero del 2013 en la población de L. olivacea en Playa Tortuga y otras playas del Pacífico (Solano, 2013).

Se ha comprobado que Playa Tortuga es efectivamente una playa de anidación de $L$. olivaceae, cuya población ha sido sujeta a una gran presión histórica por causa del ser humano, lo que ha reducido drásticamente el número de hembras que anidan en comparación a otras playas del Pacífico Sur de Costa Rica.

Patrullajes exhaustivos en una playa de poca extensión permitieron registrar a través de marcaje una muestra representativa de la fracción de la población que arriba en una temporada específica. Los individuos identificados aportaron un gran porcentaje de los neonatos reclutados a la población, por lo que la pérdida de uno de ellos en un grupo de hembras como el de Playa Tortuga podría tener un efecto mayor que en otras playas de mayor anidación.

La dinámica natural de la playa en los últimos años junto con factores de riesgo a mediano y largo plazo, como el desarrollo sin control y el turismo no sostenible, aunado a situaciones puntuales como la interacción de la especie con las pesquerías, podrían causar daños irreversibles en la población. Cabe mencionar que estos 
problemas no son exclusivos de Playa Tortuga sino que muchas de estas situaciones suceden en otras playas del litoral Pacífico de Costa Rica (Orrego, 2005).

Por tales motivos el registro de aspectos de la anidación, como el número de los eventos, las estimaciones de poblaciones de hembras que desovan, y el establecimiento de parámetros base como la distribución físico-temporal en playa de las tortugas y nidadas, resulta una herramienta útil para evidenciar fluctuaciones a lo largo del tiempo.

Basado en estos resultados se podrían ejecutar planes y acciones de mitigación de riesgos para la especie, como los programas de pesca responsable, la educación y sensibilización ambiental, planes reguladores adecuados para las zonas costeras y la ejecución de las leyes de protección del recurso marino costero.

\section{AGRADECIMIENTOS}

A Leo Whiteside, Alexia Maizel y Frank Tortoriello, por el apoyo brindado, para la ejecución del presente estudio. Se agradece al Proyecto Consolidación de Áreas Marinas Protegidas del Sistema Nacional de Áreas de Conservación, Programa de Naciones Unidas para el Desarrollo y el Global Environment Facility (GEF) por su colaboración en la publicación.

\section{RESUMEN}

En las playas de arribada de Costa Rica el comportamiento de anidación y otros aspectos de la biología de Lepidochelys olivacea han sido ampliamente estudiados, pero existe poca información acerca de las playas de anidación solitaria en especial del Pacífico Sur. Por este motivo el objetivo del presente estudio es describir los aspectos relevantes del comportamiento de la anidación de L. olivacea en Playa Tortuga, Pacífico Sur de Costa Rica, durante tres temporadas de anidación (2010, 2011, 2012). Se dividió la playa en 14 sectores de $100 \mathrm{~m}$ cada uno, a partir de esto se realizaron patrullajes todas noches durante los meses de julio a diciembre (2010) y de julio a enero $(2011,2012)$, con el fin de registrar el comportamiento de anidación de las tortugas y proteger sus nidadas. Las hembras observadas fueron identificadas mediante la aplicación de marcas metálicas en sus aletas posteriores, se registró el número de huevos, el sector de ubicación de las nidadas, la hora, datos biométricos ancho y largo curvo del caparazón (ACC-LCC), también se contabilizaron todas las nidadas efectivas (con o sin tortuga). Se logró marcar 100 hembras de L. olivacea, y se contabilizaron un total de 233 nidadas en Playa Tortuga. Se determinó una frecuencia de anidación de dos veces por temporada con un intervalo de reanidación de 14 a 20 días y para cuatro individuos se registró un período de remigración de un año. Se estimó para las tres temporadas una población total de 117 hembras y se determinó el período de agosto a setiembre como el pico de anidación. Con respecto a la biometría, se obtuvo un valor de LCC promedio de $69.91 \pm 1.05 \mathrm{~cm}$ y un valor de ACC promedio de $70.476+/-1.767 \mathrm{~cm}$. Un total de 18711 huevos fueron colectados y 9858 neonatos fueron reclutados. Se ha comprobado a partir de las tres temporadas de monitoreo que Playa Tortuga debe de ser considerada como una playa de anidación de $L$. olivacea, a pesar de que que su población actual de hembras anidantes es reducida en comparación a otras playas del Pacífico Sur. El marcaje es una herramienta útil para la caracterización de la frecuencia e intervalo de reanidación.

Palabras clave: Tortuga marina, anidación, marcaje, Pacífico Sur, población.

\section{REFERENCIAS}

Abreu-Grobois, A., \& Plotkin, P. (2008). Lepidochelys olivacea. Version 2012.2. IUCN Red List of Threatened Species. Recuperado de www.iucnredlist.org

Alvarado, J., \& Murphy, T. (2000). Periodicidad en la anidación y el Comportamiento entre anidaciones. In K. L. Eckert, K. A. Bjorndal, F. A. Abreu-Grobois \& M. Donnelly (Eds.), Técnicas de investigación y manejo para la conservación de las tortugas marinas (pp. 132-136). Pensilvania, USA: UICN/CSE Grupo de especialistas en tortugas marinas.

Ballestero, J., Aráuz, R. M., \& Rojas, R. (1998, March). Management, conservation, and sustained use of olive Ridley sea turtle eggs (Lepidochelys olivacea) in the Ostional Wildlife Refuge, Costa Rica. An 11 year review. Submitted at the XVIII Annual Symposium on Sea Turtle Biology and Conservation, Mazatlan, México.

Bolongaro, A., Márquez, A. Z., Torres, V., \& García, A. (2010). Vulnerabilidad de sitios de anidación de tortugas marinas por efectos de erosión costera en el estado de Campeche. In A. V. Botella, S. VillanuevaFragoso, J. Gutiérrez \& J. L. Rojas-Galaviz (Eds.), Vulnerabilidad de las zonas costeras mexicanas ante el cambio climático (pp. 514). México: Universidad Autónoma de Campeche.

Bolten, A. (2000). Técnicas para la medición de Tortugas Marinas. In K. L. Eckert, K. A. Bjorndal, F. A. Abreu-Grobois \& M. Donnelly (Eds.), Técnicas 
de investigación y manejo para la conservación de las tortugas marinas (pp. 126-131). Pensilvania, USA: UICN/CSE Grupo de especialistas en tortugas marinas.

Campbell, L. M. (1998). Use them or lose them? Conservation and the consumptive use of the marine turtle eggs at Ostional, Costa Rica. Environmental Conservation, 25, 305-319.

Chacón, D., Valerin, N., Cajiao, M. V., Gamboa, H., \& Marin, G. (2000). Manual para mejores prácticas de conservación de tortugas marinas en Centroamérica. Red Regional de para la Conservación de las Tortugas marinas de Centroamérica.

Chacón, D., \& Aráuz, R. (2001). Diagnóstico Regional y planificación estratégica para la conservación de las Tortugas Marinas en Centroamérica. Red Regional de para la Conservación de las Tortugas marinas de Centroamérica.

Chacón, D., Sánchez, J., Calvo. J., \& Ash, J. (2007). Manual para el manejo y la conservación de las tortugas marinas de Costa Rica; con énfasis en la operación de proyectos en playa y viveros. San José, Costa Rica: Sistema Nacional de Áreas de Conservación (SINAC), Ministerio de Ambiente y Energía (MINAE).

Chinchilla, G., \& Naranjo, J. (2010). Resumen Meteorológico Noviembre 2010 (Boletín Meteorológico Mensual del IMN). Costa Rica: Instituto Meteorológico Nacional.

Cornelius, S. E., \& Robinson, D. C. (1986). Post nesting movements of female olive ridley sea turtles tagged in Costa Rica. Vida Silvestre Neotropical, 1, 12-23.

Eckert, K., \& Beggs, J. (2006). Marcado de Tortugas Marinas. Un Manual de Métodos Recomendados (Informe Técnico No. 2). North Carolina, USA: Red de Conservación de Tortugas Marinas del Gran Caribe (WIDECAST).

Gulko, D., \& Eckert, K. (2004). Sea Turtles: An ecological guide. Honolulu, HI, USA: Mutual Publishing.

Hughes, D., \& Richard, J. (1974). The nesting of the Pacific Ridley turtle Lepidochelys olivacea on Playa Nancite, Costa Rica. Marine Biology, 24, 97-107.
Mortimer, J. A., \& Pritchard, P. C. H. (2000). Taxonomía, Morfología Externa e Identificación de las Especies. In K. L. Eckert, K. A. Bjorndal, F. A. Abreu-Grobois \& M. Donnelly (Eds.), Técnicas de investigación y manejo para la conservación de las tortugas marinas (pp. 23-44). Pensilvania, USA: UICN/CSE Grupo de especialistas en tortugas marinas.

Orrego, C. (2005). Causas antropicas y naturales en la mortalidad de las tortugas baula (Dermochelys coriacea), lora (Lepidochelys olivaceae), y verde (Chelonia mydas agassizi), en la costa pacifica de Costa Rica (Informe Programa Regional en Manejo y Conservación de Vida Silvestre para Meso América y el Caribe). Universidad Nacional, Heredia, Costa Rica.

Plotkin, P. T. (2010). Nomadic behavior of the highly migratory olive Ridley sea turtle Lepidochelys olivacea in the eastern tropical Pacific Ocean. Endangered Species Research, 13, 33-40.

Sánchez, A. (2007). Programa de Conservación, Investigación y Educación de Tortugas Marinas en la Península de Osa, Playas Carate, Rio Oro, Pejeperro y Piro (Reporte Técnico Temporada 2006). Costa Rica: Corcovado Foundation. Recuperado de: http:// www.latinamericanseaturtles.org/archivos/documentos/Osa2006.pdf

Solano, H. (2 de febrero, 2013). Estudios sobre muerte de tortugas en el Pacífico Sur revelan asfixia. La Nación. Recuperado de http://www.nacion.com/2013-02-01/ AldeaGlobal/Estudios-sobre-muerte-de-tortugas-enel-Pacifico-Sur-revelan-asfixia.aspx

Valverde, R. A., \& Gates, C. E. (2000). Estudios de Poblaciones en Playas de Arribadas. In K. L. Eckert, K. A. Bjorndal, F. A. Abreu-Grobois \& M. Donnelly (Eds.), Técnicas de investigación y manejo para la conservación de las tortugas marinas (pp. 64-69). Pensilvania, USA: UICN/CSE Grupo de especialistas en tortugas marinas.

Whinterington, B. E., \& Martin, R. E. (1996). Understanding, assessing and resolving light-pollution problems on sea turtles nesting beaches (Report TR-2). Florida, USA: Florida Marine Research Institute. 
